\title{
Technical Note: Some Properties of Splitting Criteria
}

\author{
LEO BREIMAN \\ Statistics Department, University of California, Berkeley, CA 94720
}

leo@stat.berkeley.edu

Editor: Paul Utgoff

\begin{abstract}
Various criteria have been proposed for deciding which split is best at a given node of a binary classification tree. Consider the question: given a goodness-of-split criterion and the class populations of the instances at a node, what distribution of the instances between the two children nodes maximizes the goodnessof-split criterion? The answers reveal an interesting distinction between the gini and entropy criterion.
\end{abstract}

Keywords: Trees, Classification, Splits

\section{Introduction}

There are different splitting criteria in use for growing binary decision trees. The CART program offers the choice of the gini or twoing criteria. Many other programs use the entropy criterion. Recently Fayyad (1991) and Fayyad and Irani (1990, 1992, 1993) proposed other criteria, which give improved accuracy on a number of data sets. Taylor and Silverman (1993) also explore alternative criteria, and Buntine and Niblet (1992) compare various splitting rules.

To be more specific, suppose that a class of splits $\{s\}$ is defined on the data in a node $t$. A "goodness-of-split" function $\theta(s, t)$ is defined and the best split taken as the maximizer of $\theta(s, t)$. Let there be $J$ classes numbered $1, \ldots, J$, and denote the proportions of the classes in $t$ by $\mathrm{p}=p_{1}, \ldots, p_{J}$. If $s$ sends a proportion $P_{L}$ of the $t$ population left and $P_{R}=1-P_{L}$ right, then assume

$$
\theta(s, t)=f\left(P_{L}, P_{R}, \mathbf{p}_{L}, \mathbf{p}_{R}\right)
$$

where $\mathbf{p}_{L}=\left(p_{1, L}, \ldots, p_{J, L}\right)$ is the proportion of the $J$ classes in the left node $t_{L}$ and similarly for $\mathrm{p}_{R}$.

Equivalently, for every split $s$, there are numbers $\alpha_{j}, 0 \leq \alpha_{j} \leq 1$, and $\beta_{j}=1-\alpha_{j}$ such that $P_{L}=\sum_{j} \alpha_{j} p_{j}, P_{R}=\sum \beta_{j} p_{j}, p_{j, L}=\alpha_{j} p_{j} / P_{L}, p_{j, R}=\beta_{j} p_{j} / P_{R}$ and $\theta(s, t)=$ $f(\boldsymbol{\alpha}, \boldsymbol{p})$. In practice, the set of splits is restricted, e.g. univariate, but what we explore here is the question of what happens if all possible splits are allowed. That is, over the set of all $\alpha \in[0,1]^{J}$, which $\alpha$ maximizes $\theta(s, t)$ ? We answer this question for goodnessof-split criteria generated by impurity functions (Breiman, et al., 1984). We call the split corresponding to the maximizing $\alpha$ the optimum split even though it may not be realizable in terms of splits on the input variables.

If $\boldsymbol{p}=\left(p_{1}, \ldots, p_{J}\right)$ are the node proportions, then $\phi(\boldsymbol{p})$ is an impurity function if it is convex in $p$, has a maximum when all $p_{j}$ are equal and is a minimum when one of the 
$p_{j}=1$. For $\phi(\boldsymbol{p})$ an impurity function the associated goodness-of-split is defined as

$$
\theta(s, t)=\phi(\boldsymbol{p})-P_{L} \phi\left(\boldsymbol{p}_{L}\right)-P_{R} \phi\left(\boldsymbol{p}_{R}\right) .
$$

The most commonly encountered impurity functions are the gini:

$$
\phi(p)=\sum_{j} p_{j}\left(1-p_{j}\right)
$$

and the entropy

$$
\phi(\boldsymbol{p})=-\sum_{j} p_{j} \log p_{j}
$$

Another criterion discussed in Breiman et al. (1984) (pp. 104-106) is twoing. The idea is to find that grouping of all $J$ classes into two superclasses so that considered as a two-class problem, the greatest decrease in node impurity is realized. If the gini impurity measure is used in the two class problem, then it is shown that the best twoing split at a node maximizes

$$
\theta(s, t)=\frac{P_{L} P_{R}}{4}\left[\sum_{j}\left|p_{j, L}-p_{j, R}\right|\right]^{2}
$$

and that when the split maximizing $\theta$ is used, the two superclasses are

$$
\begin{aligned}
& \mathcal{C}_{1}=\left\{j ; p_{j, L} \geq p_{j, R}\right\} \\
& \mathcal{C}_{2}=\left\{j ; p_{j, L}<p_{j, R}\right\} .
\end{aligned}
$$

For splitting criteria generated by impurity functions, our approach reveals interesting differences. For example, the optimum split for the gini criterion sends all data in the class with the largest $p_{j}$ to $t_{L}$ and all other classes to $t_{R}$. Thus the best gini splits try to produce pure nodes. But the optimal split under the entropy criterion breaks the classes up into two disjoint subsets $\mathcal{C}_{1}, \mathcal{C}_{1}^{c} \subset\{1, \ldots, J\}$ such that $\mathcal{C}_{1}$ minimizes $\left|\sum_{j \in \mathcal{C}} p_{j}-.5\right|$ among all subsets $\mathcal{C} \subset\{1, \ldots, J\}$. Thus, optimizing the entropy criterion tends to equalize the sample sizes in $t_{L}, t_{R}$. The twoing criterion also tries to equalize.

The outline is as follows: in Section 2 we show that the split optimizing $\theta(s, t)$ has the property that all $\alpha_{j}$ are zero or one. That is, no classes have parts both in $t_{L}$ and $t_{R}$. In Section 3 we find the optimal splits under the gini, entropy, and twoing measures. Section 4 gives conclusions. In particular, the results for the entropy measure suggest use of a partial look-ahead strategy.

\section{Optimal Splits Do Not Split Classes}

Let $\phi(\mathbf{x})$ be defined and twice differentiable for $\mathbf{x} \in[0,1]^{J}$. Assume that $\phi(\mathbf{x})$ is convex, i.e. the matrix $\left(\partial^{2} \phi / \partial x_{i} \partial x_{j}\right)$ is non-positive definite for all $\boldsymbol{x} \in[0,1]^{J}$. Let the impurity of $t$ be $\phi(\mathbf{p})$ and the goodness-of-split be the decrease in impurity, i.e.

$$
\theta(s, t)=\phi(\mathbf{p})-P_{L} \phi\left(\mathbf{p}_{L}\right)-P_{R} \phi\left(\mathbf{p}_{R}\right)
$$


ThEOREM 1 Let $P_{L}=\sum \alpha_{j} p_{j}, p_{j, L}=\alpha_{j} p_{j} / P_{L}, P_{R}=1-P_{L}, p_{j, R}=\left(1-\alpha_{j}\right) p_{j} / P_{R}$. Then the maximum impurity decrease over $\alpha \in[0,1]^{J}$ is achieved at a vertex of $[0,1]^{J}$.

Proof: Suppose $P_{L} \phi\left(\mathbf{p}_{L}\right)+P_{R} \phi\left(\mathbf{p}_{R}\right)$ is convex in $\alpha$. Then its minimum over $[0,1]^{J}$ is at an extreme point of $[0,1]^{J}$, i.e. a vertex. It is sufficient to show that $P_{L} \phi\left(\mathbf{p}_{L}\right)$ is convex in $\boldsymbol{\alpha}$, since $P_{R} \phi\left(\mathbf{p}_{R}\right)$ is the same function of $\boldsymbol{\beta}=\mathbf{e}-\boldsymbol{\alpha}(\mathbf{e}=(1, \ldots, 1))$ as $P_{L} \phi\left(\mathbf{p}_{L}\right)$ is of $\alpha$ and the sum of convex functions is convex.

The rest of the proof comes from using the result that

$$
\frac{\partial^{2}}{\partial \alpha_{i} \partial \alpha_{j}}\left(P_{L} \phi\left(\mathbf{p}_{L}\right)\right)=\frac{1}{P_{L}} p_{i} p_{j} \sum_{\ell, h} \phi_{\ell h}\left(\delta_{i \ell}-\frac{\alpha_{\ell} p_{\ell}}{P_{L}}\right)\left(\delta_{j h}-\frac{\alpha_{h} p_{h}}{P_{L}}\right)
$$

where

$$
\phi_{\ell h}=\left.\frac{\partial^{2} \phi(\mathrm{x})}{\partial x_{\ell} \partial x_{h}}\right|_{\mathbf{x}=\mathbf{p} L} .
$$

Equation (2.1) is derived in the Appendix. To show $P_{L} \phi\left(p_{L}\right)$ convex in $\boldsymbol{\alpha}$, it is sufficient to show that for any $J$-vector $\boldsymbol{u}$,

$$
\sum_{i j} u_{i} u_{j} \frac{\partial^{2}}{\partial \alpha_{i} \partial \alpha_{j}}\left(P_{L} \theta\left(\boldsymbol{p}_{L}\right)\right) \leq 0
$$

For any $J$-vector $\mathrm{u}$, define the $J$-vector $v$ by

$$
v_{\ell}=\sum_{i} u_{i} p_{i}\left(\delta_{i \ell}-\frac{\alpha_{\ell} p_{\ell}}{P_{L}}\right)=u_{\ell} p_{\ell}-\frac{\alpha_{\ell} p_{\ell}}{P_{L}}\left(\sum_{i} u_{i} p_{i}\right)
$$

Then

$$
\sum u_{i} u_{j} \frac{\partial^{2}}{\partial \alpha_{i} \partial \alpha_{j}}\left(P_{L} \phi\left(\mathbf{p}_{L}\right)\right)=\frac{1}{P_{L}} \sum_{\ell, n} \mathrm{v}_{\ell} \mathrm{v}_{h} \phi_{\ell h} .
$$

Since $\phi$ is convex, this last term is non-positive, and thus, $P_{L} \phi\left(\mathbf{p}_{L}\right)$ is convex in $\alpha$.

Both the gini and entropy criteria are of the form

$$
\phi(\mathbf{x})=\sum_{j} f\left(x_{j}\right)
$$

with $f(x)$ convex implying $\phi$ convex. The gini $f$ is $x(1-x)$ and entropy $f$ is $-x \log x$.

The twoing criterion is

$$
\theta(s, t)=\frac{P_{L} P_{R}}{4}\left[\sum_{j}\left|p_{j, L}-p_{j, R}\right|\right]^{2} .
$$

This is not given by a difference in impurities, so the theorem above does not directly apply. Recall that the twoing criterion is derived from dividing the classes into two superclasses, finding the best gini split in this two class problem, and then optimizing the decrease in impurity over all divisions into two superclasses. If all splits are allowed, then the above theorem implies that each optimum two class split sends all of one class to $t_{L}$ and all of the other to $t_{R}$. Thus, the best twoing split is also at a vertex of $[0,1]^{J}$. 


\section{Specific Optima}

This section answers the question of which vertex of $[0,1]^{J}$ is optimum for the entropy, gini, and twoing criteria. For the entropy measure, we want to maximize

$$
P_{L} \sum_{j} p_{j, L} \log p_{j, L}+P_{R} \sum_{j} p_{j, R} \log p_{j, R} .
$$

For a given vertex, let $\mathcal{C}_{0}=\left\{j ; \alpha_{j}=0\right\}, \mathcal{C}_{1}=\left\{j ; \alpha_{j}=1\right\}$. The above expression becomes

$$
\begin{gathered}
P_{L} \sum_{j \in \mathcal{C}_{1}}\left(p_{j} / P_{L}\right) \log \left(p_{j} / P_{L}\right)+P_{R} \sum_{j \in \mathcal{C}_{0}}\left(p_{j} / P_{R}\right) \log \left(p_{j} / P_{R}\right) \\
=\sum_{j} p_{j} \log p_{j}-P_{L} \log P_{L}-P_{R} \log P_{R} .
\end{gathered}
$$

The optimum vertex maximizes

$$
-P_{L} \log P_{L}-P_{R} \log P_{R}
$$

So at the best vertex $\left|P_{L}-.5\right|$ is minimized.

With the gini measure, the best vertex minimizes

$$
\begin{gathered}
P_{L} \sum p_{j, L}\left(1-p_{j, L}\right)+P_{R} \sum p_{j, R}\left(1-p_{j, R}\right)= \\
P_{L} \sum_{j \in \mathcal{C}_{1}}\left(p_{j} / P_{L}\right)\left(1-p_{j} / P_{L}\right)+P_{R} \sum_{j \in \mathcal{C}_{0}}\left(p_{j} / P_{R}\right)\left(1-\left(p_{j} / P_{R}\right) .\right.
\end{gathered}
$$

Equivalently, choose that vertex which maximizes

$$
\frac{1}{P_{L}} \sum_{j \in \mathcal{C}_{1}} p_{j}^{2}+\frac{1}{P_{R}} \sum_{j \in \mathcal{C}_{0}} p_{j}^{2}
$$

PROPOSITION Let $p_{i}=\max _{j}\left(p_{j}\right)$. Then the best gini vertex sends all of class $i$ to $t_{L}$ and the remainder to $t_{R}$.

The proof of this proposition involves some algebraic manipulation and is deferred to the appendix. Finally, note that on any vertex, the twoing measure (2) equals $P_{L} P_{R} / 4$. Thus, the best vertex minimizes $\left|P_{L}-.5\right|$.

\section{Discussion and Conclusions}

The above shows the difference between the best splits selected using the gini criterion versus the entropy and twoing criteria. The gini prefers splits that put the largest class into one pure node, and all others into the other. Entropy and twoing put their emphasis on 
balancing the sizes at the two children nodes. These theoretical conclusions get support in the simulations in Breiman et. al (1984) (see pp. 111).

In problems with a small number of classes, all criteria should produce similar results. The differences appear in data where $J$ is larger. Here, high up in the tree, gini may produce splits that are too unbalanced. On the other hand, the above results show a disturbing facet of the entropy and twoing criterion, i.e. a lack of uniqueness. If $J$ is moderate to large, there are usually many vertices such that $P_{L} \simeq .5$. For instance, in a little simulation, we took $J=10$ and selected the $\left\{p_{j}\right\}$ to be uniform random numbers, suitably normalized. On the average, for each set of $\left\{p_{j}\right\}$ about 40 vertices gave $P_{L}$ values between .49 and .51 with 4 vertices such that $.499 \leq P_{L} \leq .501$. These vertices often differed in the distribution of both the larger and smaller $p_{j}$ values.

Since many vertices have similar goodness-of-split values, selecting the best split is a bit arbitrary. Which split is best depends on the future evolution of the tree. This suggests that use of the entropy or twoing criteria be combined with a limited two step look-ahead. For instance, one could set an integer $N$, and for each of the $N$ best splits of a node $t$ compute the total decrease in impurity following the splits of $t_{L}$ into $t_{L L}, t_{L R}$ and $t_{R}$ into $t_{R L}, t_{R R}$. Then use the best of the $N$. One must take care to ensure that if some of the $N$ splits are on the same variable, they are sufficiently different.

\section{Appendix}

\section{Derivation of (2.1)}

Let $x_{j}=\alpha_{j} p_{j} / P_{L}$. Then for any $g(\boldsymbol{x})$

$$
\begin{aligned}
\frac{\partial g(x)}{\partial \alpha_{j}} & =\sum_{\ell} \frac{\partial g}{\partial x_{\ell}}\left(-\frac{\alpha_{\ell} p_{\ell} p_{j}}{P_{L}^{2}}+\delta_{\ell j} \frac{p_{j}}{P_{L}}\right) \\
& =\frac{p_{j}}{P_{L}}\left(\frac{\partial g}{\partial x_{j}}-\sum_{\ell} \frac{\partial g}{\partial x_{\ell}} x_{\ell}\right)
\end{aligned}
$$

Using the notation $\phi_{i}=\partial \phi / \partial x_{i}, \phi_{i j}=\partial^{2} \phi / \partial x_{i} \partial x_{j}$ and applying (A.1) gives

$$
\frac{\partial}{\partial \alpha_{j}}\left(P_{L} \phi(\boldsymbol{x})\right)=p_{j}\left[\phi+\phi_{j}-\sum_{\ell} \phi_{\ell} x_{\ell}\right] .
$$

Take $H(\boldsymbol{x})$ to be the term in brackets in the above equation and note that

$$
\frac{\partial H(\boldsymbol{x})}{\partial x_{i}}=\phi_{i j}-\sum_{\ell} \phi_{i \ell} x_{\ell} .
$$

Using (A.1) again

$$
\frac{\partial}{\partial \alpha_{i}}\left(p_{j} H\right)=\frac{p_{i} p_{j}}{P_{L}}\left(\frac{\partial H}{\partial x_{i}}-\sum_{h} \frac{\partial H}{\partial x_{h}} x_{h}\right)
$$




$$
\begin{aligned}
& =\frac{p_{i} p_{j}}{P_{L}}\left[\phi_{i j}-\sum_{\ell} \phi_{i \ell} x_{\ell}-\sum_{h} \phi_{h j} x_{h}+\sum_{\ell, h} \phi_{h \ell} x_{h} x_{\ell}\right] \\
& =\frac{p_{i} p_{j}}{P_{L}} \sum_{\ell, h} \phi_{\ell h}\left(\delta_{i \ell}-x_{\ell}\right)\left(\delta_{j h}-x_{h}\right)
\end{aligned}
$$

Proof of the proposition: For any set of indices $\mathcal{C} \subset\{1, \ldots, J\}$, let $Q(\mathcal{C})=\sum_{j \in \mathcal{C}} p_{j}^{2}$, $P(\mathcal{C})=\sum_{j \in \mathcal{C}} p_{j}$, and $\lambda(\mathcal{C})=Q(\mathcal{C}) / P(\mathcal{C})$. We want to maximize $G(\mathcal{C})=\lambda(\mathcal{C})+\lambda\left(\mathcal{C}^{c}\right)$. If $\mathcal{C}$ maximizes $G$, so does $\mathcal{C}^{c}$. Take as $\mathcal{C}$ whichever one satisfies $\lambda(\mathcal{C}) \geq \lambda\left(\mathcal{C}^{c}\right)$. Let $p_{i}=\max \left(p_{j}, j \in \mathcal{C}\right)$, and take $\mathcal{C}_{1}=\mathcal{C}-\{i\}$. We will show that

$$
G(\{i\}) \geq G(\mathcal{C})
$$

so that a maximizer of $G$ sends all cases in one class to one child node, and all other classes to the other child.

The inequality A.2 follows from the identity

$$
G(\{i\})-G(\mathcal{C})=\frac{P\left(\mathcal{C}_{1}\right)}{p_{i}\left(1-p_{i}\right)}\left[\left(1-p_{i}\right) \lambda(\mathcal{C})-p_{i} \lambda\left(\mathcal{C}^{c}\right)-\left(1-2 p_{i}\right) \lambda\left(\mathcal{C}_{1}\right)\right]
$$

This identity can be derived from the simpler identity

$$
G(\{i\})=\frac{1}{p_{i}}\left[\lambda(\mathcal{C}) p(\mathcal{C})-Q\left(\mathcal{C}_{1}\right)\right]+\frac{1}{1-p_{i}}\left[\lambda\left(\mathcal{C}^{c}\right) P\left(\mathcal{C}^{c}\right)+Q\left(\mathcal{C}_{1}\right)\right]
$$

Subtracting $G(\mathcal{C})$ from A.4 and simplifying gives A.3. Suppose first that $p_{i} \geq 1 / 2$. Then to prove A. 2 its sufficient to show that

$$
\left(1-p_{i}\right) \lambda(\mathcal{C}) \geq p_{i} \lambda\left(\mathcal{C}^{c}\right)
$$

For any subset $\mathcal{D}$ of indices

$$
\begin{aligned}
Q(\mathcal{D}) & \leq(P(\mathcal{D}))^{2} \\
\Rightarrow \lambda(\mathcal{D}) & \leq P(\mathcal{D}) .
\end{aligned}
$$

Now

$$
\left(1-p_{i}\right) \lambda(\mathcal{C})=\left(1-p_{i}\right) \frac{Q(\mathcal{C})}{P(\mathcal{C})} \geq \frac{\left(1-p_{i}\right) p_{i}^{2}}{P(\mathcal{C})}
$$

and $\lambda\left(\mathcal{C}^{c}\right) \leq P\left(\mathcal{C}^{c}\right)=1-P(\mathcal{C})$. Hence A.5 will follow from

$$
\left(1-p_{i}\right) p_{i} \geq P(\mathcal{C})(1-P(\mathcal{C}))
$$

The expression $x(1-x)$ is decreasing for $x \geq 1 / 2$. Since $P(\mathcal{C})>p_{i}$, A. 6 is true.

Now assume $p_{i} \leq 1 / 2$. Then A.2 again follows from showing that the term in brackets in $\mathrm{A} .3$ is non-negative. Rewrite this term as

$$
\left(1-2 p_{i}\right) \lambda(\mathcal{C})+p_{i}\left(\lambda(\mathcal{C})-\lambda\left(\mathcal{C}^{c}\right)\right)-\left(1-2 p_{i}\right) \lambda\left(\mathcal{C}_{1}\right)
$$


By assumption, this is greater than or equal to

$$
\left(1-2 p_{i}\right)\left(\lambda(\mathcal{C})-\lambda\left(\mathcal{C}_{1}\right)\right)
$$

Since

$$
\lambda(\mathcal{C})-\lambda\left(\mathcal{C}_{1}\right)=p_{i} \frac{\left(p_{i} P(\mathcal{C})-Q(\mathcal{C})\right)}{P(\mathcal{C})\left(P(\mathcal{C})-p_{i}\right)}
$$

and $Q(\mathcal{C}) \leq p_{i} P(\mathcal{C})$, the proof of A.2 is complete, and $G(\{i\})$ is a maximizer of $G(\mathcal{C})$.

Now we show that if $p_{i} \leq p_{j}$, then $G(\{i\}) \leq G(\{j\})$. This follows from the identity

$$
G(\{j\})-G(\{i\})=\left(p_{j}-p_{i}\right)\left[\left(1-p_{i}-p_{j}\right)^{2}+\sum_{h \neq i, j} p_{h}^{2}\right] /\left(1-p_{i}\right)\left(1-p_{j}\right)
$$

resulting from straightforward algebra.

\section{References}

Breiman, L., Friedman, J., Olshen, R., \& Stone, C. (1984). "Classification and Regression Trees," Wadsworth.

Buntine, W. \& Niblett, T. (1992). "A further comparison of splitting rules for decision tree induction," Machine Learning 8, 75-85.

Fayyad, U.M. (1991). "On the induction of decision trees for multiple concept learning," Ph.D Thesis, Department of Electrical Engineering and Computer Science, University of Michigan, Ann Arbor, Michigan.

Fayyad, U.M. \& Irani, R.B. (1990). "What should be minimized in a decision tree"? Proc. 8th National Conf. on AI, AAAI-90, 749-754, MIT Press.

Fayyad, V.M. \& Irani, R.B. (1992). "The attribute selection problem in decision tree generation," Proc. 10th National Conf. on AI, AAAI-92, 104-110, MIT Press.

Fayyad, U.M. \& Irani, R.B. (1993). "Multi-interval discretization of continuous valued attribute for classification learning," Proc. 13th International Joint Conf. on AI, 1022-1027, Morgan Kaufmann.

Taylor, P.C. \& Silverman, B.W. (1993). "Block diagrams and splitting criteria for classification trees" Statistics and Computing, V 3, p. 147-161.

Received April 29, 1994

Accepted August 26, 1994

Final Maunscript October 2, 1995 